OPEN ACCESS

Edited by:

Benjamin Gourion,

UMR2594 Laboratoire des

Interactions Plantes-Microorganismes

(LIPM), France

Reviewed by:

Mark James Banfield,

John Innes Centre (BBSRC),

United Kingdom

Mehdi Kabbage

University of Wisconsin-Madison,

United States

*Correspondence:

Alberto P. Macho

alberto.macho@sibs.ac.cn

${ }^{t}$ These authors have contributed

equally to this work.

Specialty section:

This article was submitted to

Plant Microbe Interactions,

a section of the journal

Frontiers in Plant Science

Received: 25 July 2017

Accepted: 19 October 2017

Published: 07 November 2017

Citation:

Wei Y, Sang Y and Macho AP (2017)

The Ralstonia solanacearum Type III

Effector RipAY Is Phosphorylated in Plant Cells to Modulate Its

Enzymatic Activity.

Front. Plant Sci. 8:1899.

doi: 10.3389/fpls.2017.01899

\section{The Ralstonia solanacearum Type III Effector RipAY Is Phosphorylated in Plant Cells to Modulate Its Enzymatic Activity}

\author{
Yali Wei ${ }^{1,2 t}$, Yuying Sang ${ }^{1 \dagger}$ and Alberto P. Macho ${ }^{1 * \dagger}$ \\ 1 Shanghai Center for Plant Stress Biology, CAS Center for Excellence in Molecular Plant Sciences, Shanghai Institutes of \\ Biological Sciences, Chinese Academy of Sciences, Shanghai, China, ${ }^{2}$ University of Chinese Academy of Sciences, \\ Beijing, China
}

Most bacterial pathogens subvert plant cellular functions using effector proteins delivered inside plant cells. In the plant pathogen Ralstonia solanacearum, several of these effectors contain domains with predicted enzymatic activities, including acetyltransferases, phosphatases, and proteases, among others. How these enzymatic activities get activated inside plant cells, but not in the bacterial cell, remains unknown in most cases. In this work, we found that the $R$. solanacearum effector RipAY is phosphorylated in plant cells. One phosphorylated serine residue, S131, is required for the reported gamma-glutamyl cyclotransferase activity of RipAY, responsible for the degradation of gamma-glutamyl compounds (such as glutathione) inside host cells. Accordingly, non-phosphorylable mutants in S131 abolish RipAY-mediated degradation of glutathione in plant cells and the subsequent suppression of plant immune responses. In this article, we examine our results in relation to the recent reports on the biochemical activities of RipAY, and discuss the potential implications of phosphorylation in plant cells as a mechanism to modulate the enzymatic activity of RipAY.

Keywords: effector phosphorylation, effector activation, post-translational modification, plant immunity, plant disease

\section{INTRODUCTION}

Many bacterial plant pathogens secrete dozens of proteins inside plant cells using a type-III secretion system (T3SS). These proteins (type III-secreted effectors; T3Es) are key determinants of the interaction between pathogen and host, contributing to the alteration of plant cellular processes to promote infection (Deslandes and Rivas, 2012; Macho, 2016). T3E activities can also be perceived by resistant plants, leading to the activation of immune responses (Khan et al., 2016). The bacterial pathogen Ralstonia solanacearum causes the devastating bacterial wilt disease in numerous hosts, constituting an important threat to agriculture (Mansfield et al., 2012), but is also an emerging system for the study of T3Es (Deslandes and Genin, 2014). Strains from $R$. solanacearum express more than 70 different effectors (Peeters et al., 2013), and the T3Sdependent secretion or translocation of most of them has been experimentally validated (Cunnac et al., 2004; Mukaihara et al., 2010). Remarkably, many of these effectors contain domains with 
predicted enzymatic activities, including acetyltransferases, phosphatases, and proteases, among others (Dean, 2011; Peeters et al., 2013). After delivery inside plant cells, these proteins have the potential to associate with plant molecules in a specific or unspecific manner, catalyzing their modification to promote bacterial proliferation and the development of disease (Dean, 2011; Macho and Zipfel, 2015). This raises the intriguing question of how the enzymatic activities of these bacterial effectors are maintained in an inactive state in the bacterial cell, but become active inside host cells after their delivery through the T3SS. High degree of substrate specificity could be a potential mechanism employed by bacterial enzymes to prevent activity toward bacterial substrates. However, strict substrate specificity may be difficult to achieve in many cases, or impossible in scenarios where the substrate molecule is also present in bacterial cells. Specific modification of T3Es inside eukaryotic cells has been proposed as a mechanism to explain the selective activation of T3E enzymatic activities after their delivery into host cells. Accordingly, in recent years, several T3Es from bacterial pathogens have been shown to undergo activation through interaction with host factors or posttranslational modifications (PTMs) inside plant cells, such as phosphorylation, ubiquitination, or lipidations (Popa et al., 2016).

The R. solanacearum T3E RipAY is present in all the $R$. solanacearum strains sequenced to date (Peeters et al., 2013), and contains a ChaC domain, exhibiting gammaglutamyl cyclotransferase (GGCT) activity (Fujiwara et al., 2016; Mukaihara et al., 2016). RipAY employs this GGCT activity to degrade the redox buffer glutathione (and potentially other gamma-glutamyl compounds) in plant cells, causing a suppression of plant immune responses (Fujiwara et al., 2016; Mukaihara et al., 2016; Sang et al., 2016). Interestingly, although the degradation of plant glutathione seems a powerful strategy for bacterial pathogens to subvert plant physiological functions, no other T3E has been reported to exhibit GGCT activity. A potential danger caused by the bacterial production of a GGCT enzyme is the degradation of bacterial gamma-glutamyl compounds, and therefore GGCT activity must be selectively activated after delivery inside host cells. Eukaryotic thioredoxins have been shown to contribute to the activation of RipAY GGCT activity in vitro, which is otherwise not detectable after RipAY purification from bacterial cells (Fujiwara et al., 2016; Mukaihara et al., 2016). However, the mechanism of thioredoxin-mediated activation of RipAY remains unknown. In this work, we report that RipAY is phosphorylated in plant cells. Site-directed mutagenesis of the phosphorylated residues indicates that a serine residue is required for the RipAY-mediated degradation of glutathione and suppression of immune responses triggered by bacterial elicitors, although it is not required for the reported interaction with plant thioredoxins. We discuss the possible interpretations of this result, raising questions and offering new perspectives for the study of phosphorylation in plant cells as a potential mechanism to modulate enzymatic activities of RipAY and other R. solanacearum T3Es.

\section{MATERIALS AND METHODS}

\section{Plant Material, Growth Conditions, and Transient Expression Assays}

Nicotiana benthamiana plants used in this study were grown in an environmentally controlled growth room at $22^{\circ} \mathrm{C}$ with a $16 \mathrm{~h}$ photoperiod. Agrobacterium-mediated transient expression was performed as described ( $\mathrm{Li}, 2011)$. Bacterial suspensions were adjusted to a final $\mathrm{OD}^{600}$ of 0.5 before infiltration.

\section{Chemicals}

The flg22 peptide (sequence from Pseudomonas syringae pv. tomato DC3000; TRLSSGLKINSAKDDAAGLQIA) was purchased from Abclonal, United States. Sequencing-grade modified trypsin was purchased from Promega (Madison, WI, United States). The Nanosep centrifugal filter units with Omega membrane (MWCO $10 \mathrm{kDa}$ ) were bought from Pall Corporation (New York, NY, United States). All other chemicals were purchased from Sigma-Aldrich (St. Louis, MO, United States) unless otherwise stated.

\section{Immunoprecipitation and LC-MS/MS Analysis}

Immunoprecipitation assays and Western blots were performed as described previously (Sang et al., 2016). Briefly, total $N$. benthamiana proteins were extracted and incubated with GFP-trap beads (ChromoTek, Germany). After washing, IPed proteins were stripped from the beads and separated on precast SDS-PAGE gels (Bio-Rad, Hercules, CA, United States). Western blot was performed using the anti-FLAG (Sigma) and anti-GFP (Abiocode, Agoura Hills, CA, United States) primary antibodies, respectively. Blots were stained with Coomassie Brilliant Blue to verify equal loading. Protein digestion, liquid-chromatography coupled to tandem mass-spectrometric analysis and peptide identification were performed as described (Sang et al., 2016).

\section{ROS and Glutathione Measurements}

The measurement of the flg22-triggered ROS burst is detailed in Sang and Macho (2017). Total cellular glutathione was measured using the Glutathione Assay Kit (Sigma-Aldrich) according to the manufacturer's instructions, as described (Sang et al., 2016).

\section{Constructs and Site-Directed Mutagenesis}

Constructs for transient expression of RipAY and thioredoxins have been described before (Sang et al., 2016). New RipAY mutant variants were generated as described previously (Sang et al., 2016), using the primers listed in Supplementary Table S1.

\section{Confocal Microscopy and Image Processing}

Leaves from 4- to 5-week-old $N$. benthamiana plants were infiltrated with Agrobacterium to induce the expression of RipAY-GFP or the indicated mutant versions. Two days after inoculation, RipAY-GFP subcelullar localization was observed in 
pavement epidermal cells. GFP imaging was performed on an inverted laser scanning confocal microscope (Leica TCS SP8) equipped with LEICA LAS X image acquisition software. The green fluorescent protein (GFP) was excited by a $488 \mathrm{~nm}$ argon laser at a strength of $20 \%$ and detected at 501-528 $\mathrm{nm}(\mathrm{Em})$.

\section{RESULTS}

\section{RipAY Is Phosphorylated in Plant Cells}

In order to identify RipAY residues post-translationally modified in plant cells, we previously performed Agrobacterium-mediated transient expression of RipAY (cloned from the GMI1000 strain; phylotype 1) fused to a green-fluorescent protein tag (RipAYGFP) in Nicotiana benthamiana cells (Sang et al., 2016). GFP immunoprecipitation (IP) followed by liquid chromatography and tandem-mass spectrometry (LC-MS/MS) allowed us to identify several RipAY peptides containing phosphorylated serine (S) and threonine $(\mathrm{T})$ residues (Figure 1A and Supplementary Figure S1). Among these residues, S131 and S251 are located within the ChaC domain (Supplementary Figure S1A), and are conserved in RipAY sequences from several $R$. solanacearum strains representative from different phylotypes (Figure 1A and Supplementary Figure S2). An additional phosphorylated residue, S32, was only conserved in strains from the phylotype I, although RipAY sequences from other strains present serine residues in the position 30 or 31 (Supplementary Figure S2).

\section{S131 Is Important for RipAY Activity in Planta}

RipAY-GFP transiently expressed in N. benthamiana is able to suppress the rapid generation of reactive oxygen species (ROS) after treatment of plant tissues with the immune elicitor flg22 (Sang et al., 2016). In order to determine whether the potential phosphorylated residues found in the LC-MS/MS assays are important for RipAY immune suppression activity, we performed single or multiple site-directed mutagenesis of these residues, substituting them to the non-phosphorylable residue alanine (A). As shown in the Figure 1B, the S131A mutation abolished RipAY ability to suppress flg22-triggered ROS burst (Figure 1B). Strikingly, although the single S32A mutation did not affect RipAY suppression of flg22-triggered ROS, expression of the double S32A/S131A mutant consistently increased this response in comparison with GFP-expressing control tissues (Figure 1B).

It has been previously shown that the ability of RipAY to suppress immune responses is associated to the degradation of plant glutathione through its GGCT activity (Mukaihara et al., 2016; Sang et al., 2016). To test whether the potential phosphorylated residues are important for RipAY catalytic activity, we measured glutathione content in plant tissues expressing RipAY or the different mutants carrying the mentioned amino-acid substitutions. N. benthamiana tissues expressing RipAY showed a dramatic reduction of glutathione content (10-15\%) compared with GFP-expressing control tissues (Figure 1C). Mutants containing the S131A mutation were partially impaired in glutathione degradation in comparison with wild-type RipAY (50-60\% compared to control tissues; Figure 1C). Besides S131A, additional mutations in other phosphorylated residues did not significantly affect RipAY ability to degrade plant glutathione (Figure 1C).

We next tested whether S131 phosphorylation could contribute to the activation of RipAY function. Mutation of serine to aspartic acid (D) sometimes resembles the addition of a phosphate group on a serine residue, mimicking a phosphorylation event. However, a S131D mutation abolished RipAY ability to suppress flg22-triggered ROS burst (Supplementary Figure S3).

The mutagenesis of the phosphorylated residues did not affect RipAY accumulation (Supplementary Figure S4A). As we reported before, we consistently detected a double band corresponding to RipAY in Western blot assays (Sang et al., 2016; Supplementary Figure S4), which may be caused by PTMs of the protein. This double band became more obvious when using acrylamide gels containing PhosTag, which binds phosphorylated residues, slowing down the migration of phosphorylated proteins (Kinoshita et al., 2006; Supplementary Figure S4B), suggesting that this band-shift may be caused by phosphorylation. Interestingly, the S32A mutation altered the ratio between these bands, increasing the concentration of the fast-migrating band (Supplementary Figure S4), suggesting that phosphorylation in S32 accounts for most RipAY phosphorylation, or that phosphorylation of this site is required for the subsequent phosphorylation of others.

\section{The S32A and S131A Mutations Do Not Affect RipAY Subcellular Localization or Interaction with Plant Thioredoxins}

Protein phosphorylation can affect protein-protein interactions and the subcellular localization of phosphorylated proteins. We previously reported that RipAY-GFP localizes at the cytoplasm and nucleus of $N$. benthamiana cells, and associates with several thioredoxins (TRXs) in planta (Sang et al., 2016). Association with plant TRXs has been shown to contribute to RipAY activation in vitro (Fujiwara et al., 2016; Mukaihara et al., 2016). Since the S32A mutation altered RipAY bandshift and S131A abolished RipAY activity, we tested whether these mutations affect the subcellular localization of RipAYGFP or its association with plant thioredoxins. Confocal microscopy showed that transiently expressed RipAY-GFP localizes at the cytoplasm and nucleus of $N$. benthamiana cells, and the S131A or S32AS131A mutations did not affect this subcellular localization (Figure 2A). Similarly, none of these mutations altered the reported RipAY-GFP association with NbTRX09 and NbTRX15 in Co-IP assays (Figure 2B).

\section{DISCUSSION AND PERSPECTIVES}

Upon transient expression in $N$. benthamiana cells, we were able to detect phosphorylation in several RipAY peptides (Figure 1A). Mutagenesis of the phosphorylated residues to 
A

\begin{tabular}{|c|c|c|c|}
\hline Site & Peptide & $\begin{array}{c}\text { Best } \\
\text { Mascot } \\
\text { score }\end{array}$ & Conservation \\
\hline S32 & (K)NGpSPPRR(A) & 36.8 & - \\
\hline S131 & (K)GLNYVPVFGYLpSLR(Y) & 36 & + \\
\hline S251 & (R)ELLDENDLIHPVPATPPAGTTGLAGSATAGPATPLPSPK(K) & 99.3 & + \\
\hline
\end{tabular}

B

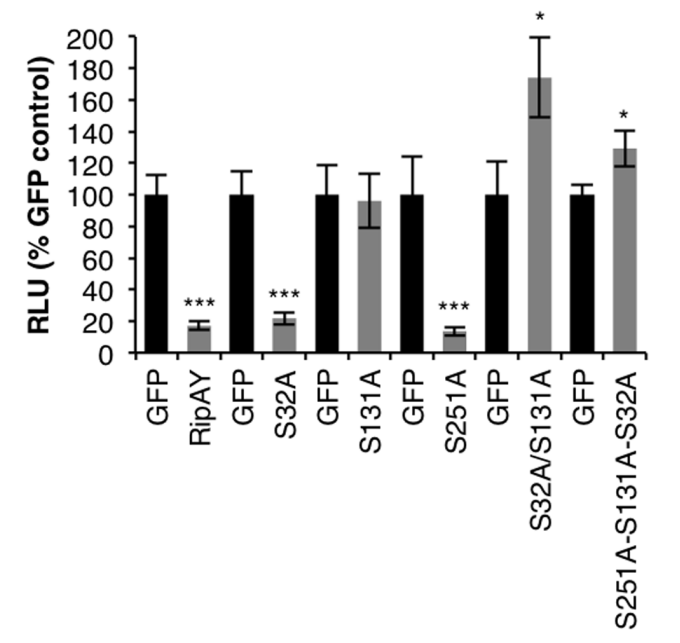

C

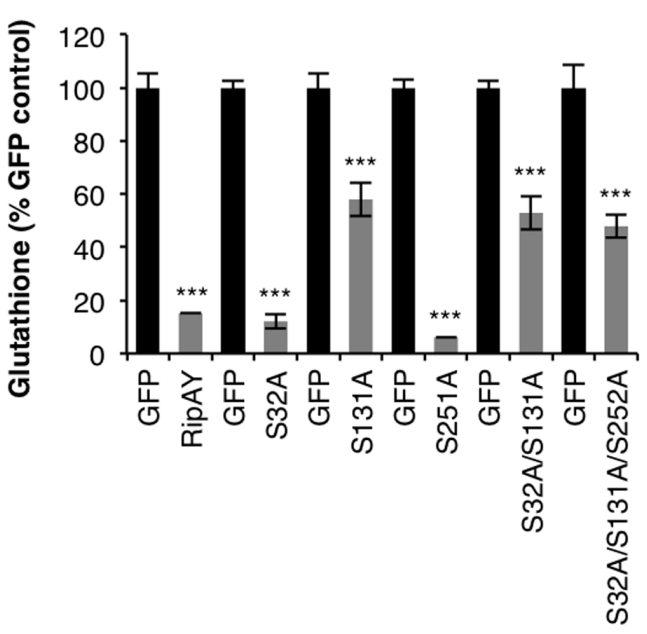

FIGURE 1 | Relevance of phosphorylation for RipAY activity in plant cells. (A) Tryptic peptides containing phosphorylated serine or threonine residues found after IP+LC-MS/MS of RipAY-GFP expressed in N. benthamiana. Only peptides with a Mascot ion score above 35 were considered. Lowercase $\mathrm{p}$ indicates the phosphorylation site. The conservation of the phosphorylated sites in RipAY sequences from $R$. solanacearum strains from different phylotypes is indicated (see Supplementary Figure S2 for details). (B) A. tumefaciens was used to induce the transient expression of RipAY-GFP (or the indicated mutant variants) in half of a $N$. benthamiana leaf and GFP in the other half. The graph shows total oxidative burst triggered by $100 \mathrm{nM}$ flg22 in N. benthamiana tissues 2.5 days post-inoculation (dpi) with $A$. tumefaciens, and measured in a luminol-based assay as relative luminescence units (RLUs). Values are average $\pm \mathrm{SE}$ ( $n=8$ ). (C) Total glutathione content in $N$. benthamiana tissues 2.5 dpi with $A$. tumefaciens inducing the expression of GFP, RipAY-GFP (or the indicated mutant variants). Values are average \pm SE $(n=3)$. Asterisks indicate significant differences compared to the corresponding GFP control $\left(* * * P<0.001 ;{ }^{*} P<0.05\right)$. The experiments were repeated three times with similar results.

alanine indicated that S32A alters the putative phosphorylationassociated bandshift of RipAY, and mutations in S131 abolish its function. Interestingly, although S32 seems to be the major site responsible for the RipAY bandshift (Supplementary Figure S4B), mutations in that residue did not affect the RipAY-mediated degradation of glutathione or suppression of immune-related ROS burst (Figure 1). Therefore, it remains unclear whether phosphorylation in S32 (and the potential subsequent phosphorylation of other sites) is not relevant for RipAY activity or required for other activities not measured in our assays. Moreover, although the S131A mutation completely abolished the ability of RipAY to suppress the immune-related ROS burst, it only partially compromised its ability to degrade glutathione (Figure 1C), indicating that S131 has a quantitative contribution, but is not fully required, for the GGCT activity of RipAY.

We failed to induce activation of RipAY in planta by substituting S131 to an aspartic acid residue. It is still possible that phosphorylation of S131 is important for activity, but S131D cannot mimic phosphorylation. Of note, phosphorylation is a dynamic process, and the addition/removal of a phosphate group may need to be regulated in spatiotemporal manner. In such situations, the substitutions to negatively charged amino acids might not mimic phosphorylation, but rather result in loss of function, as it has been observed for phospho-mimic mutants before (Oh et al., 2009).

Importantly, S131 has been reported to be present within the putative substrate-binding site of the RipAY ChaC domain (Fujiwara et al., 2016). A S131A mutation partially suppressed RipAY ability to degrade glutathione in vitro and abolished RipAY inhibition of yeast growth (Fujiwara et al., 2016). It is possible that both phospho-dead and phospho-mimic mutations disrupt the RipAY ChaC substrate-binding site and thus disrupt activity. This raises the possibility that the observed in planta phosphorylation of S131 is not biologically relevant, although we should not rule out that plant kinases may be actively phosphorylating this site in an attempt to disrupt RipAY activity. This has been shown for Pto kinase, which abolishes AvrPtoB E3 ubiquitin ligase activity by phosphorylating an essential threonine residue in the catalytic domain of AvrPtoB (Ntoukakis et al., 2009). In that case, also, both phospho-dead and phospho-mimic 
A

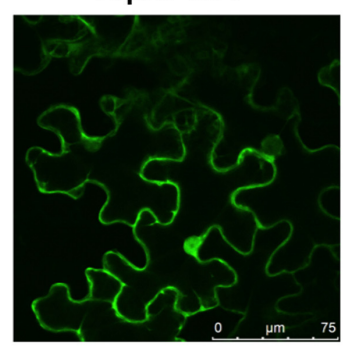

S131A/S32A-GFP
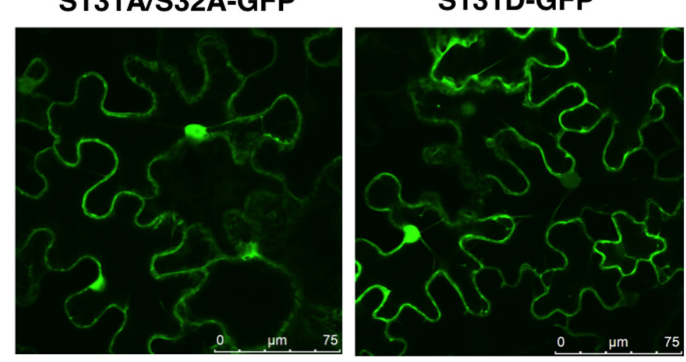

B

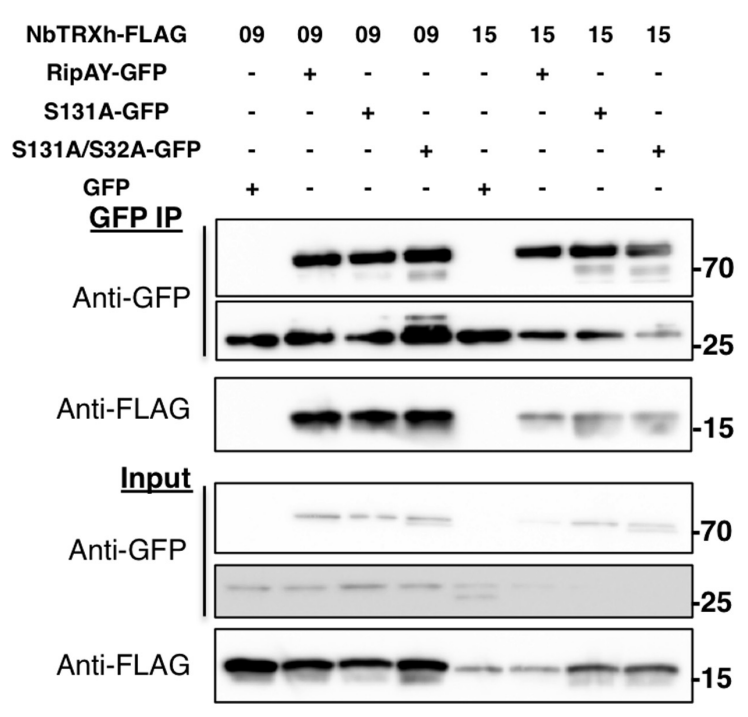

FIGURE 2 | The S32A and S131A mutations do not affect RipAY subcellular localization or interaction with plant thioredoxins. (A) Representative images of RipAY-GFP and the indicated mutant variants transiently expressed in $N$. benthamiana leaf pavement cells. Size bars $(75 \mu \mathrm{m})$ are shown for reference. (B) GFP or RipAY-GFP were co-expressed with the indicated h-type thioredoxins in N. benthamiana before immunoprecipitation using GFP-trap beads. Immunoblots were analyzed using anti-GFP or anti-FLAG antibody. Molecular weight $(\mathrm{kDa})$ marker bands are indicated for reference. A band corresponding to approximately $27 \mathrm{kDa}$ can be detected in RipAY samples, and this most likely reflects the cleavage of the GFP tag in the process of protein overexpression and extraction. The experiments were repeated twice with similar results.

mutations in the phosphorylated residue (S450A or S450D, respectively) abolish the catalytic E3 ligase of the effector (Ntoukakis et al., 2009). In the case of transiently expressed wild-type RipAY in $N$. benthamiana cells, we can find peptides phosphorylated in S131, but this transient expression is still able to cause the degradation of glutathione and suppression of immune responses. Therefore, either this mechanism of plantmediated RipAY inactivation is not predominant in natural conditions, or a portion of RipAY may be inactivated by phosphorylation, but effector overexpression in our assays may allow enough active RipAY to degrade glutathione. The PTM of important residues in the active sites of T3Es could constitute a powerful strategy for plants to inactivate their catalytic activities. Being highly conserved sites subjected to strong positive selection (due to their importance for the catalytic activity), it would be difficult for the pathogen to alter their sequence in order to avoid inactivation in resistant hosts.

The presence of TRXs has been shown to activate RipAY GGCT activity in vitro, although the underlying mechanism is still unknown (Fujiwara et al., 2016; Mukaihara et al., 2016). Therefore, we sought to determine whether S131 phosphorylation would be required for binding to TRXs as a mechanism to contribute to RipAY activation. However, RipAY S32A/S131A still associated with TRXs in planta (Figure 2), suggesting that phosphorylation of these sites is not required for TRX binding. Interestingly, although TRXs catalyze the reduction of disulphide bonds of target proteins, a mutation in the sole cysteine $(\mathrm{C})$ residue of RipAY (C333S) did not abolish TRX binding or any of RipAY activities in vivo and in vitro (Fujiwara et al., 2016; Sang et al., 2016). Recombinant RipAY purified from Escherichia coli cells shows thioredoxin-dependent GGCT activity in vitro (Fujiwara et al., 2016; Mukaihara et al., 2016). This suggests that RipAY phosphorylation by plant kinases is not required for GGCT activity in vitro. However, we have often observed several phosphorylated peptides in recombinant effector proteins purified from E. coli in conditions of overexpression, and this is a common phenomenon observed during the purification of active recombinant kinases from E. coli cells. Therefore, in conditions of overexpression, effectors can be phosphorylated by $E$. coli kinases and thus be purified in phosphorylated form, enabling in vitro reactions that require the phosphorylation of effector residues. Alternatively, it is possible that RipAY phosphorylation modulates the interaction of RipAY with substrates or other host factors required for its activity in plant cells, but dispensable for in vitro assays toward generic substrates. Structural biology approaches will be key to determine the potential relevance of the phosphorylated residues and the interaction with host factors for the activation of the catalytic activity of RipAY.

Phosphorylation has been found in an increasing number of T3Es from different plant pathogens (Popa et al., 2016), such as the Pseudomonas syringae T3E HopQ1, which contains a domain with predicted ADP-ribosyltransferase activity. HopQ1 is phosphorylated in plant cells, and this is required for its interaction with 14-3-3 proteins, which determine its subcellular localization and molecular activity (Giska et al., 2013; Li et al., 2013). R. solanacearum contains a HopQ1 ortholog, named RipB, which has conserved 14-3-3-binding sites and associates with 14-3-3 proteins in plant cells (Giska et al., 2013). 
Additionally, we have found phosphorylated peptides in several other $R$. solanacearum T3Es with predicted enzymatic activities after transient expression in plant tissues followed by protein extraction, co-IP and LC-MS/MS analysis (data not shown). These findings suggest that phosphorylation may be an extended mechanism for the modulation of the catalytic activity of $R$. solanacearum T3Es in plant cells, although the validation of this hypothesis will require the specific biochemical characterization of each T3E independently. Furthermore, the development of more sensitive proteomic approaches will help determining whether these phosphorylation events take place in natural conditions during the infection and whether other T3Es injected simultaneously inside plant cells may play a role in these modifications.

Using the RipAY amino acid sequence, a search in the Scansite 3 server $^{1}$ predicted that S32 and S251 are phosphorylated by kinases from the proline-dependent serine/threonine kinase group, such as cyclin-dependent kinases. However, we were not able to detect such kinases among RipAY interactors in our IP+LC-MS/MS approach in planta (Sang et al., 2016). To date, only two plant kinases, Pto and PIK1, have been found to phosphorylate T3Es (AvrPtoB and AvrBsT, respectively; Ntoukakis et al., 2009; Kim et al., 2014). The identification of plant kinases involved in the phosphorylation of $R$. solanacearum T3Es will be essential to fully understand their biochemical activation, and will be an important step toward the biotechnological application of this fundamental knowledge to the development of disease-resistant plants. Given the transient nature of kinase-substrate interactions, this constitutes a challenge for future studies.

\section{AUTHOR CONTRIBUTIONS}

YW, YS, and AM designed experiments, performed experiments, and analyzed data. AM wrote the paper.

\section{FUNDING}

This work was funded by the Shanghai Center for Plant Stress Biology (Chinese Academy of Sciences), the National Natural

\footnotetext{
${ }^{1}$ http://scansite3.mit.edu
}

\section{REFERENCES}

Cunnac, S., Boucher, C., and Genin, S. (2004). Characterization of the cisacting regulatory element controlling HrpB-mediated activation of the type III secretion system and effector genes in Ralstonia solanacearum. J. Bacteriol. 186, 2309-2318.

Dean, P. (2011). Functional domains and motifs of bacterial type III effector proteins and their roles in infection. FEMS Microbiol. Rev. 35, 1100-1125. doi: 10.1111/j.1574-6976.2011.00271.x

Deslandes, L., and Genin, S. (2014). Opening the Ralstonia solanacearum type III effector tool box: insights into host cell subversion mechanisms. Curr. Opin. Plant Biol. 20, 110-117. doi: 10.1016/j.pbi.2014.05.002

Deslandes, L., and Rivas, S. (2012). Catch me if you can: bacterial effectors and plant targets. Trends Plant Sci. 17, 644-655. doi: 10.1016/j.tplants.2012.06.011
Science Foundation of China (grant 31571973), and the Chinese 1000 Talents Program.

\section{ACKNOWLEDGMENTS}

We thank Rosa Lozano-Durán for critical reading and suggestions to this manuscript, Mitsuaki Tabuchi for helpful discussions, and Xinyu Jian for technical and administrative assistance during this work. We thank the PSC Cell Biology Core facility for assistance with confocal microscopy, and YiMin She and Hong Ni from the PSC Proteomics facility for their collaboration in the LC-MS/MS analysis.

\section{SUPPLEMENTARY MATERIAL}

The Supplementary Material for this article can be found online at: https://www.frontiersin.org/articles/10.3389/fpls.2017.01899/ full\#supplementary-material

FIGURE S1 | RipAY is phosphorylated in several serine residues. (A) Diagram of RipAY indicating the ChaC domain (responsible for the GGCT activity) in red and the observed phosphorylation sites. E216 indicates the catalytic glutamic acid residue essential for GGCT activity. (B) Representative mass spectra of the observed phosphorylated peptides indicated in the Figure 1A.

FIGURE S2 | Amino acid sequence alignment showing the conservation of the phosphorylated residues among RipAY from different $R$. solanacearum strains. The strains are representative from the different $R$. solanacearum phylotypes: phylotype 1: FJAT91, SD54, YC45, FQY4, GMI1000; phylotype 2: UW551 and Molk2; phylotype 3: CMR15; phylotype 4: Psi07. The phosphorylated residues identified in this work in RipAY from GMl1000 are marked with an asterisk

FIGURE S3 | The S131D mutation abolishes RipAY suppression of flg22-triggered ROS. A. tumefaciens was used to induce the transient expression of RipAY-GFP (or the S131D mutant) in half of a $N$. benthamiana leaf and GFP in the other half. The graph shows total oxidative burst triggered by $100 \mathrm{nM}$ flg22 in

$N$. benthamiana tissues 2.5 days post-inoculation (dpi) with $A$. tumefaciens, and measured in a luminol-based assay as relative luminescence units (RLUs). Values are average $\pm \mathrm{SE}(n=8)$. Asterisks indicate significant differences compared to the corresponding GFP control at $P<0.001$. The experiments were repeated at least three times with similar results.

FIGURE S4 | All the RipAY mutant variants accumulate to wild-type levels. (A) Western blot assay showing the accumulation of the mutant variants shown in the Figures 1, 2 and Supplementary Figure S3. (B) Western blot assay using a Phos-Tag PAGE gel. Immunoblots were analyzed using anti-GFP antibody. Molecular weight $(\mathrm{kDa})$ marker bands are indicated for reference.

Fujiwara, S., Kawazoe, T., Ohnishi, K., Kitagawa, T., Popa, C., Valls, M., et al. (2016). RipAY, a plant pathogen effector protein, exhibits robust gammaglutamyl cyclotransferase activity when stimulated by eukaryotic thioredoxins. J. Biol. Chem. 291, 6813-6830. doi: 10.1074/jbc.M115.678953

Giska, F., Lichocka, M., Piechocki, M., Dadlez, M., Schmelzer, E., Hennig, J., et al. (2013). Phosphorylation of HopQ1, a type III effector from Pseudomonas syringae, creates a binding site for host 14-3-3 proteins. Plant Physiol. 161, 2049-2061. doi: 10.1104/pp.112.209023

Khan, M., Subramaniam, R., and Desveaux, D. (2016). Of guards, decoys, baits and traps: pathogen perception in plants by type III effector sensors. Curr. Opin. Microbiol. 29, 49-55. doi: 10.1016/j.mib.2015.10.006

Kim, N. H., Kim, D. S., Chung, E. H., and Hwang, B. K. (2014). Pepper suppressor of the G2 allele of skp1 interacts with the receptor-like cytoplasmic kinasel and type III effector AvrBsT and promotes the hypersensitive cell death response in 
a phosphorylation-dependent manner. Plant Physiol. 165, 76-91. doi: 10.1104/ pp. 114.238840

Kinoshita, E., Kinoshita-Kikuta, E., Takiyama, K., and Koike, T. (2006). Phosphatebinding tag, a new tool to visualize phosphorylated proteins. Mol. Cell. Proteomics 5, 749-757. doi: 10.1074/mcp.T500024-MCP200

Li, W., Yadeta, K. A., Elmore, J. M., and Coaker, G. (2013). The Pseudomonas syringae effector HopQ1 promotes bacterial virulence and interacts with tomato 14-3-3 proteins in a phosphorylation-dependent manner. Plant Physiol. 161, 2062-2074. doi: 10.1104/pp.112.211748

Li, X. (2011). Infiltration of Nicotiana benthamiana protocol for transient expression via Agrobacterium. Bio Protocol Bio101:e95.

Macho, A. P. (2016). Subversion of plant cellular functions by bacterial typeIII effectors: beyond suppression of immunity. New Phytol. 210, 51-57. doi: $10.1111 /$ nph.13605

Macho, A. P., and Zipfel, C. (2015). Targeting of plant pattern recognition receptortriggered immunity by bacterial type-III secretion system effectors. Curr. Opin. Microbiol. 23C, 14-22. doi: 10.1016/j.mib.2014.10.009

Mansfield, J., Genin, S., Magori, S., Citovsky, V., Sriariyanum, M., Ronald, P., et al. (2012). Top 10 plant pathogenic bacteria in molecular plant pathology. Mol. Plant Pathol. 13, 614-629. doi: 10.1111/j.1364-3703.2012. 00804.x

Mukaihara, T., Hatanaka, T., Nakano, M., and Oda, K. (2016). Ralstonia solanacearum type III effector RipAY is a glutathione-degrading enzyme that is activated by plant cytosolic thioredoxins and suppresses plant immunity. MBio 7:e00359-16. doi: 10.1128/mBio.00359-16

Mukaihara, T., Tamura, N., and Iwabuchi, M. (2010). Genome-wide identification of a large repertoire of Ralstonia solanacearum type III effector proteins by a new functional screen. Mol. Plant Microbe Interact. 23, 251-262. doi: 10.1094/ MPMI-23-3-0251

Ntoukakis, V., Mucyn, T. S., Gimenez-Ibanez, S., Chapman, H. C., Gutierrez, J. R., Balmuth, A. L., et al. (2009). Host inhibition of a bacterial virulence effector triggers immunity to infection. Science 324, 784-787. doi: 10.1126/science. 1169430

Oh, M. H., Wang, X., Kota, U., Goshe, M. B., Clouse, S. D., and Huber, S. C. (2009). Tyrosine phosphorylation of the BRI1 receptor kinase emerges as a component of brassinosteroid signaling in Arabidopsis. Proc. Natl. Acad. Sci. U.S.A. 106, 658-663. doi: 10.1073/pnas.0810249106

Peeters, N., Carrere, S., Anisimova, M., Plener, L., Cazale, A. C., and Genin, S. (2013). Repertoire, unified nomenclature and evolution of the type III effector gene set in the Ralstonia solanacearum species complex. BMC Genomics 14:859. doi: 10.1186/1471-2164-14-859

Popa, C. M., Tabuchi, M., and Valls, M. (2016). Modification of bacterial effector proteins inside eukaryotic host cells. Front. Cell. Infect. Microbiol. 6:73. doi: 10.3389/fcimb.2016.00073

Sang, Y., and Macho, A. P. (2017). Analysis of PAMP-triggered ROS burst in plant immunity. Methods Mol. Biol. 1578, 143-153. doi: 10.1007/978-1-49396859-6_11

Sang, Y., Wang, Y., Ni, H., Cazale, A. C., She, Y. M., Peeters, N., et al. (2016). The Ralstonia solanacearum type III effector RipAY targets plant redox regulators to suppress immune responses. Mol. Plant Pathol. doi: 10.1111/mpp.12504 [Epub ahead of print].

Conflict of Interest Statement: The authors declare that the research was conducted in the absence of any commercial or financial relationships that could be construed as a potential conflict of interest.

Copyright (c) 2017 Wei, Sang and Macho. This is an open-access article distributed under the terms of the Creative Commons Attribution License (CC BY). The use, distribution or reproduction in other forums is permitted, provided the original author(s) or licensor are credited and that the original publication in this journal is cited, in accordance with accepted academic practice. No use, distribution or reproduction is permitted which does not comply with these terms. 